\title{
Dengue Myocarditis Complicated by COVID-19: A Case Report on Dual Viral Infection
}

\author{
Samiksha Gupta ${ }^{1}$, Shreya Arora ${ }^{1}$, Monica Gupta ${ }^{1}$, Gautam Jesrani ${ }^{1}$ \\ 1. General Medicine, Government Medical College and Hospital, Chandigarh, IND
}

Corresponding author: Gautam Jesrani, jesranigautam@gmail.com

\begin{abstract}
During the ongoing pandemic of coronavirus disease 2019 (COVID-19), it is crucial for clinicians to have an insight into the emerging co-infections. As the dengue virus (DENV) and severe acute respiratory syndrome coronavirus 2 (SARS-CoV-2) have related symptomatology, a high index of suspicion is required for prompt diagnosis of concurrent infections involving these two pathogens, especially in the areas endemic for tropical diseases, i.e., dengue fever (DF), malaria, enteric fever, chikungunya, leptospirosis, etc. In this report, we present the case of a middle-aged man from Northern India, who had DF with myocarditis, and was simultaneously found to have COVID-19 co-infection. The patient was managed as per the COVID-19 protocol and had a favorable outcome.
\end{abstract}

Categories: Internal Medicine, Infectious Disease

Keywords: acute undifferentiated febrile illness, co-infection, covid-19, dengue fever, myocarditis

\section{Introduction}

Novel coronavirus disease 2019 (COVID-19), caused by severe acute respiratory syndrome coronavirus 2 (SARS-CoV-2), has resulted in a lethal global pandemic [1]. It exhibits varied clinical manifestations, potentially affecting every organ system, although fever remains a common symptom [2]. In India, dengue virus (DENV) infection, transmitted by Aedes aegypti, is one of the most common causes of acute undifferentiated febrile illness (AUFI) with endemicity, usually during the monsoon and post-monsoon seasons. The symptom profile consists of mild non-specific symptoms such as fever, malaise, and vomiting, making the clinical recognition of the disease laborious [3]. In rare cases, myocarditis can also be seen in the disease course of dengue fever (DF). Concurrent infection of COVID-19 and dengue myocarditis is rarely described in the current literature, and in this report, we describe one such presentation. Additionally, we review and describe the overlapping symptomatology in such co-infections, which is imperative for clinicians to recognize in clinical practice.

Review began $07 / 27 / 2021$ Review ended 08/02/2021 Published 08/10/2021

(c) Copyright 2021

Gupta et al. This is an open access article distributed under the terms of the Creative Commons Attribution License CC-BY 4.0., which permits unrestricted use, distribution, and reproduction in any medium, provided the original author and source are credited.

\section{Case Presentation}

A 65-year-old man with no known comorbidities in the past presented to the emergency department with a history of high-grade fever and chills for four days, which was relieved with antipyretic medication. He also had a history of intermittent episodes of the passage of reddish urine, lasting for two days, which was not associated with abdominal pain or dysuria. At presentation, he had non-productive intermittent bouts of cough but denied any exposure to COVID-19 or tuberculosis patients. On examination, the patient had a temperature of $38.5^{\circ} \mathrm{C}$, a regular pulse rate of 48 beats/minute, blood pressure of 90/64 mmHg, respiratory rate of 18 /minute, and was maintaining an oxygen saturation of $97 \%$ on room air. He also had a generalized erythematous rash over the abdomen and lower limbs (Figure 1). The cardiovascular and respiratory examination did not reveal any abnormality. His electrocardiogram demonstrated sinus bradycardia (Figure 2), but the chest radiograph revealed no cardiomegaly or lung pathology on presentation. 


\section{Cureus}

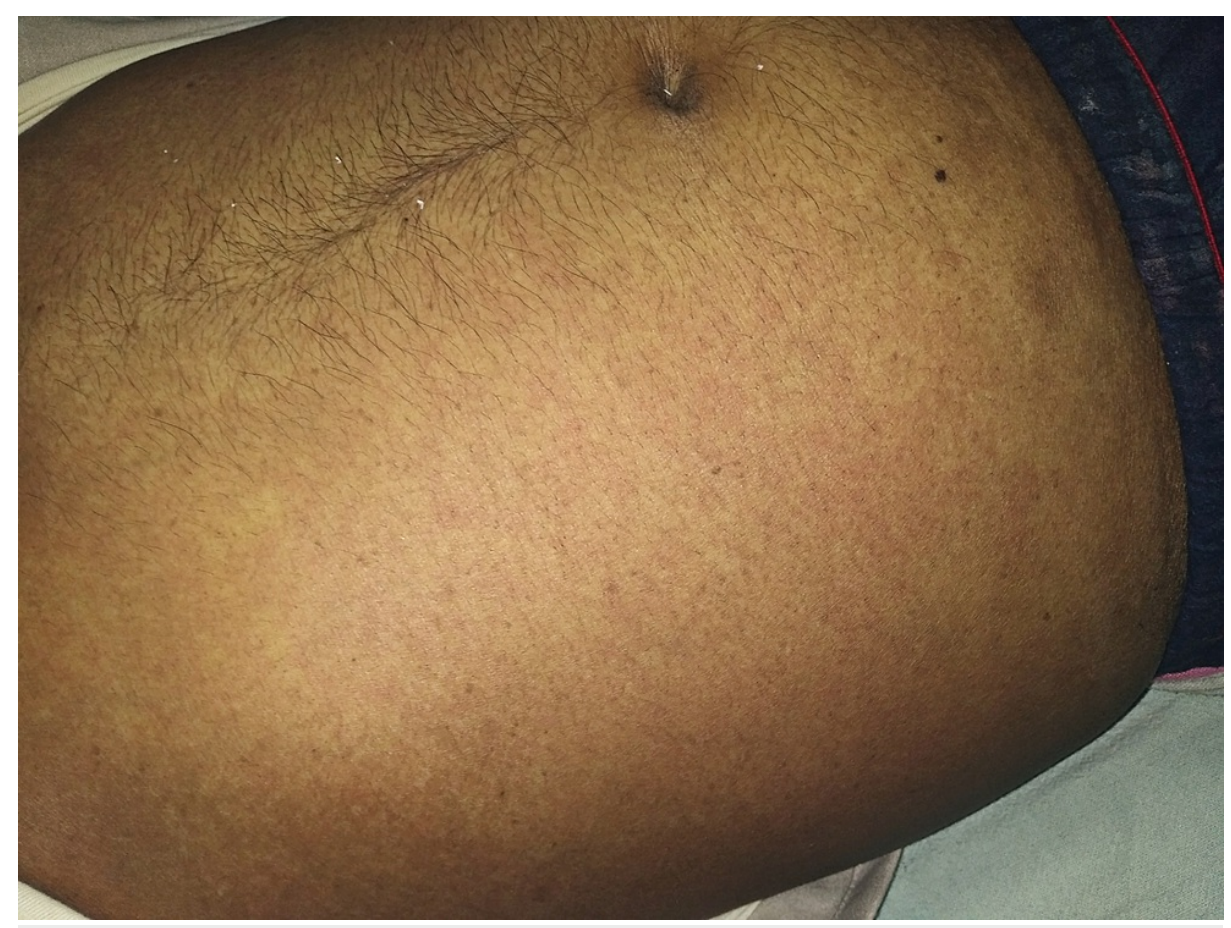

FIGURE 1: Skin rashes on the abdomen of the patient on presentation

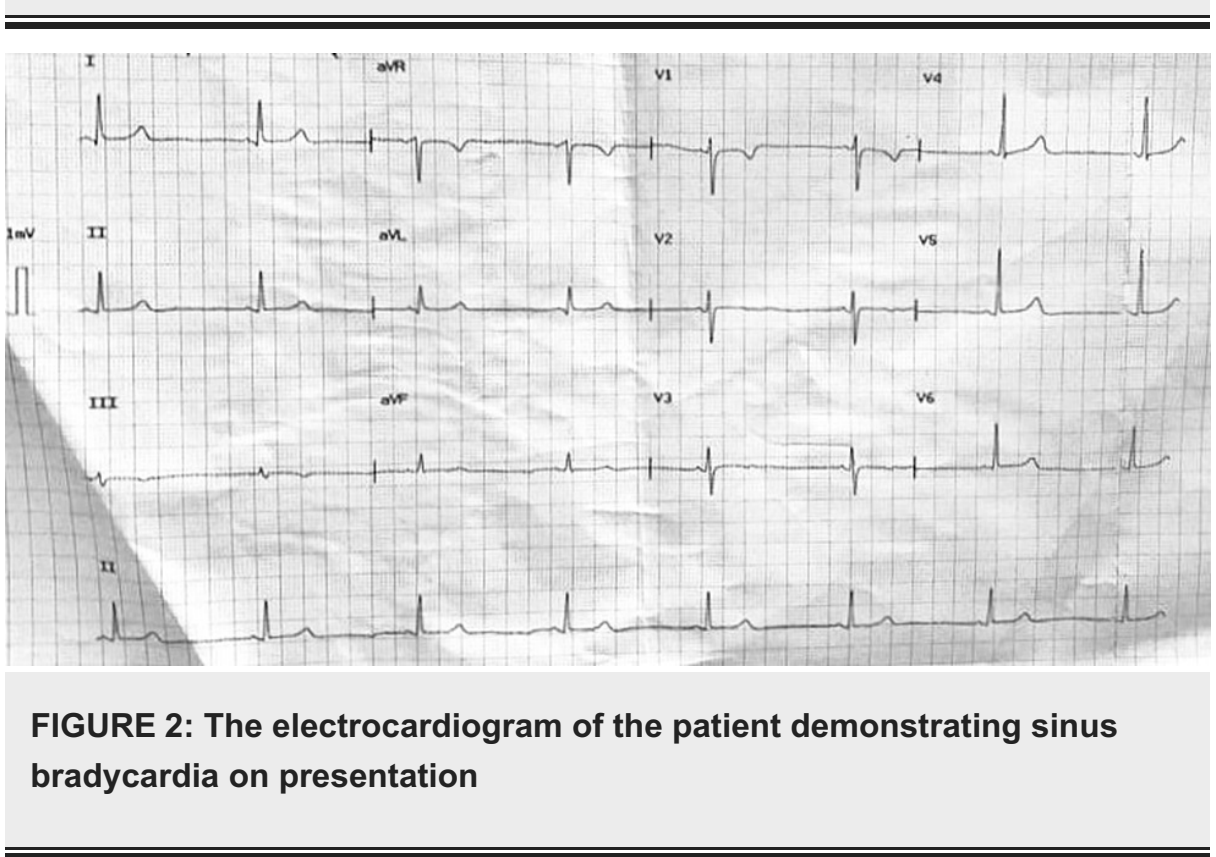

On investigations, his complete blood profile revealed a total leucocyte count of $2.3 \times 10^{9} / \mathrm{L}$ with $55 \%$ neutrophils and $40 \%$ lymphocytes and thrombocytopenia of $20 \times 10^{9} / \mathrm{L}$ (Table 1 ). In light of the thrombocytopenia, the patient was evaluated for malaria, scrub typhus, leptospirosis, enteric fever, and DF. DENV serology for immunoglobulin M (IgM) came out to be positive with test levels of 0.72 optical density (OD, cut-off value: 0.31 ) while DENV NS-1 antigen was negative by enzyme-linked immunoassay. Thrombocytopenic measures were advised (i.e., to avoid brushing teeth or straining and no intramuscular injection) along with intravenous fluid therapy. Due to relative bradycardia, the possibility of viral myocarditis was considered. His qualitative troponins were negative; however, creatine phosphokinase-MB (CPK-MB) was considerably high, i.e., $72 \mathrm{U} / \mathrm{L}$ (cut off value: $<25$ ), and N-terminal pro-B type natriuretic peptide (NT-proBNP) was $1560 \mathrm{pg} / \mathrm{mL}$ (cut off for age: $<900 \mathrm{pg} / \mathrm{mL}$ ). 2D-echocardiography showed normal left ventricular systolic function, grade-I diastolic dysfunction, and preserved ejection fraction of $64 \%$. Regarding hematuria evaluation, his ultrasound of the renal system did not reveal any anatomical discrepancy or stones within the pelvicalyceal system and therefore it was attributed to severe thrombocytopenia. 


\section{Cureus}

\begin{tabular}{|c|c|c|c|}
\hline Investigations & Normal range & Day 2 & Day 13 \\
\hline Hemoglobin (g/dL) & 12.0-16.0 & 13.0 & 13.4 \\
\hline Total leucocyte count (x 109/L) & $4.0-11.0$ & 2.3 & 5.7 \\
\hline Neutrophil-lymphocyte ratio & $1-3$ & 1.4 & 1.8 \\
\hline Platelet count (x 109/L) & $150-450$ & 20 & 178 \\
\hline Aspartate transaminase (AST) (U/L) & $5-40$ & 187 & 54 \\
\hline Alanine transaminase (ALT) (U/L) & $5-35$ & 46 & 40 \\
\hline Creatine phosphokinase-MB (U/L) & $<25$ & 72 & 31 \\
\hline C-reactive protein (CRP) (mg/L) & $0-5$ & 49 & 12 \\
\hline Procalcitonin (PCT) (ng/mL) & $<0.05$ & 0.13 & 0.03 \\
\hline D-dimer ( $\mu \mathrm{g} / \mathrm{mL})$ & $<0.5$ & 0.8 & 0.4 \\
\hline Lactate dehydrogenase (LDH) (U/L) & $235-470$ & 569 & 480 \\
\hline Interleukin-6 (pg/mL) & 4-12 & 12.2 & 5.0 \\
\hline Ferritin (ng/mL) & $22-322$ & 453 & 327 \\
\hline
\end{tabular}

TABLE 1: Important blood investigations of the patient during the hospital course

On the fifth day of hospitalization, he developed breathlessness with a respiratory rate of 26 /minute and a saturation of $90 \%$ on room air following which a repeat chest radiograph was performed, which suggested bilateral heterogeneous opacity in lower zones (left > right) and the left middle zone (Figure 3). He was managed with oxygen supplementation through a venturi mask and had a normal oxygen saturation thereafter. High-resolution CT (HRCT) of the chest was done, which was suggestive of bilateral ground-glass opacities (left > right) and a severity index of 13/25 (Figure 4). In light of the ongoing pandemic and local protocol, the patient was tested via nasopharyngeal swab by reverse transcriptase-polymerase chain reaction (RT-PCR) for COVID-19, and he was confirmed positive for SARS-CoV-2 virus. 


\section{Cureus}

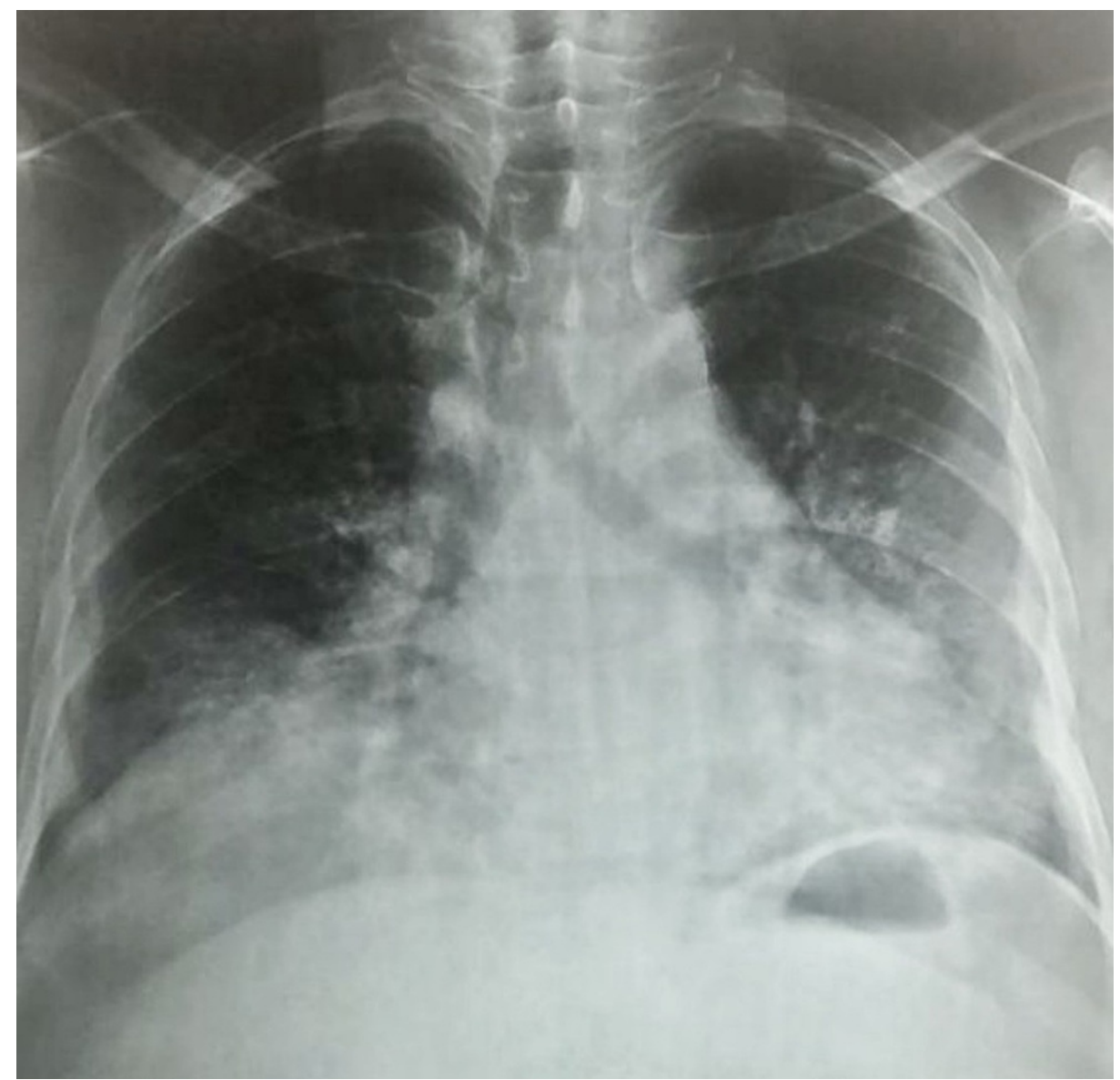

FIGURE 3: Subsequent chest X-ray of the patient demonstrating consolidation in the left middle zone and bilateral lower zone 


\section{Cureus}

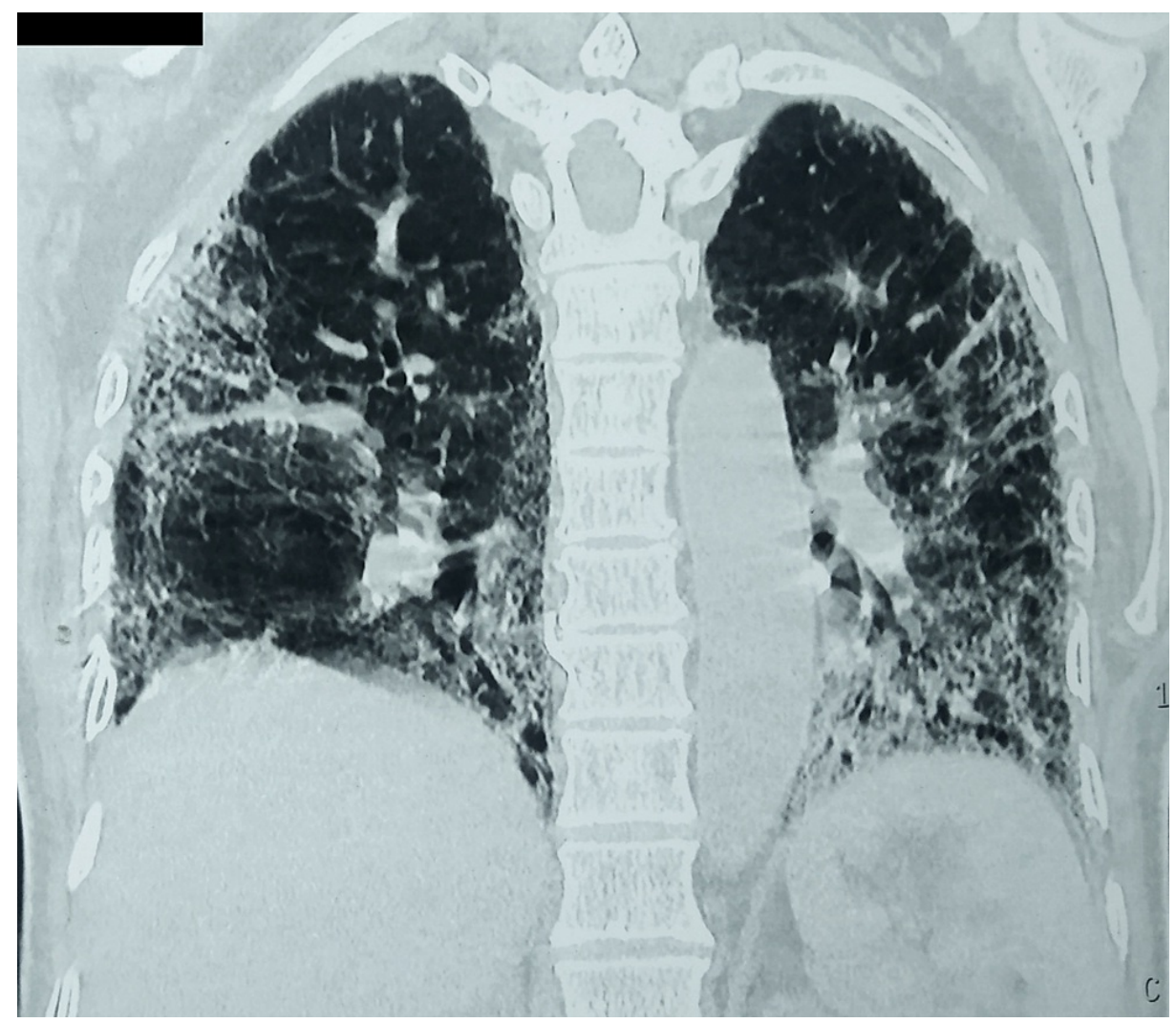

\section{FIGURE 4: Coronal section of the high-resolution CT of the chest demonstrating bilateral ground-glass opacities}

СТ: computed tomography

Only intravenous steroids $(0.1 \mathrm{mg} / \mathrm{kg}$ dexamethasone for seven days) could be given since anticoagulation was omitted due to thrombocytopenia. From day three onwards, the patient had gradual improvement in the heart rate, platelet count, and continued to have stable blood pressure. The patient was discharged successfully after 17 days in the hospital isolation facility with no residual symptoms on follow-up.

\section{Discussion}

Numerous concerns have been raised regarding the co-infection of COVID-19 with endemic illnesses such as $\mathrm{DF}$, scrub typhus, enteric fever, and leptospirosis. One of them pertains to similar presenting complaints like fever, malaise, headache, abdominal pain, or vomiting. Uncertainty and delay in diagnosis usually ensue due to the confounding clinical picture and, additionally, related to laboratory investigations for leucopenia and thrombocytopenia. Such co-infections add an additional burden to the already over-worked healthcare system in the present pandemic, especially in developing countries [4]. In our case, the patient presented with AUFI, fulfilling the laboratory criteria for DF. He subsequently tested positive for COVID-19 disease through nasopharyngeal swab by RT-PCR, prompted by the new-onset breathlessness.

Since the COVID-19 pandemic is still ongoing in Asia, which is a DENV endemic zone, co-infection of DENV and SARS-CoV-2 virus could be tricky, thereby leading to delay in the diagnosis. Although both are ribonucleic acid (RNA) viruses, they have varied pathophysiology and target organ involvement but eventually lead in the similar direction of cytokine release, resulting in dysfunction in the integrity of the vascular endothelium leading to vasculopathy, coagulopathy, and capillary leak [5]. The phenomenon of antibody-dependent enhancement (ADE) has been described for both viruses, resulting in acceleration in the degree of infection and incidence of complications [6]. Various animal studies have also shown a possible role of the angiotensin-II converting enzyme (ACE) and angiotensin in the pathophysiology of viruses such as DENV, SARS-CoV-2, and H7N9 influenza [7].

The characteristics of both the viruses in terms of epidemiological and clinical presentation are shown in Table 2 [8]. A study by Yue et al. showed a huge overlap between clinical spectrums of both diseases, with fever and headache being the most common symptoms in both [2]. Our patient had presented with fever and bleeding manifestation with no respiratory and cardiovascular symptoms at presentation or no exposure to COVID-19, and hence the possibility for a tropical illness was significant. This calls for a high index of suspicion to test for other causes in the setting of one, especially in endemic areas during monsoon and 
post-monsoon seasons. Other than non-specific signs and symptoms, which present an unpredictable influence on the severity and clinical outcome of the patient, the co-infection presents with diagnostic challenges too. Serious precautions taken to prevent the spread of transmission of COVID-19 in a febrile patient can cause a potentially dangerous delay in the diagnosis of DF. Furthermore, the COVID-19 pandemic has led to global shortages in certain laboratory reagents. Since the molecular diagnosis of both COVID-19 and DENV requires the same extraction kits and enzymes, the depletion of stocks has escalated [9]. Misleading results due to cross-reaction between these two viruses have been reported in previous studies, demonstrating that antibodies to DENV envelope hypothetically binds to "receptor binding motif (RBM)" of the spike protein of SARS-CoV-2, with certain interactions diverting the binding of angiotensinconverting enzyme (ACE-2) receptor to RBM [10].

\section{Incubation \\ period \\ 2. Signs and symptoms}

Characteristics Dengue fever

\section{COVID-19}

$\sim 14$ days, median of 4-5 days from symptom onset

Febrile phase: fever, headache with eye pain, myalgia, nausea, vomiting, rash, and leukopenia

Mild to moderate

Severe illness

With warning signs: abdominal pain or tenderness, persistent vomiting, clinical fluid accumulation, mucosal bleeding, lethargy, restlessness, and liver enlargement

DF with any of the following: plasma leakage leading to shock, fluid accumulation with respiratory distress, and severe bleeding with thrombocytopenia

Severe organ impairment such as liver disease with elevated transaminases, or meningoencephalitis with impaired consciousness

Age: infant; second DENV

3. Risk factors for infection, chronic medical severe illness conditions including diabetes, asthma, or heart disease
Fever or chills, headache, myalgia, fatigue, sore throat, cough, shortness of breath, loss of taste or smell, rhinorrhoea, nausea, vomiting, and diarrhea

Median time to dyspnea ranged from 5-8 days, the median time to acute respiratory distress syndrome (ARDS) ranged from 8-12 days, and the median time to ICU admission ranged from 10-12 days. Signs and symptoms of severe illness can include dyspnea, hypoxia, respiratory failure, shock, and multiorgan system dysfunction
Age $>65$, underlying conditions like cardiovascular disease, diabetes, chronic respiratory disease, hypertension, prior stroke, liver disease, obesity, chronic lung disease, chronic kidney disease undergoing dialysis, or immunocompromised status (i.e., poorly controlled HIV infection, undergoing cancer treatment, using corticosteroids, and smoking)

\section{TABLE 2: Characteristic features of dengue fever and COVID-19}

COVID-19: coronavirus disease 2019; HIV: human immunodeficiency virus; DENV: dengue virus; ICU: intensive care unit

Apart from posing a diagnostic dilemma, the co-infection of DENV and COVID-19 also leads to some therapeutic quandaries. Hemodynamic instability in the case of DF requires fluid administration, but it should be done cautiously due to increased chances of developing pulmonary edema and worsening of oxygenation with COVID-19 infection. COVID-19 infection has been associated with increased chances of thrombosis, which requires the administration of low molecular-weight heparin (LMWH). This, however, puts the patient at risk of life-threatening bleeding manifestations due to thrombocytopenia as a result of DENV infection, especially with a platelet count of $<1 \mathrm{lakh} / \mathrm{mm}^{3}$. The latest guidelines recommend close monitoring of D-dimer levels and platelet count if LMWH has to be given. Steroids have been shown to have a beneficial effect in COVID-19, with no negative repercussions for DF. Tocilizumab, antivirals, and other supportive management can be continued for COVID-19 as per the guidelines.

\section{Conclusions}


Dual viral infection of COVID-19 and DENV is not uncommon; it is a demanding condition, which requires a high index of suspicion. The symptoms may be non-specific in most of the cases and an individual can be asymptomatic till late in the disease course. The treating physician should be familiar with these clinical scenarios. Preventive measures like social distancing and cough etiquette implementation should be coupled with vector control and the use of insect repellents inside the hospital too. A concerted effort should be undertaken to prevent, diagnose, and treat this co-infection using vigilant measures, especially in endemic areas during rainy and post-rainy seasons.

\section{Additional Information \\ Disclosures}

Human subjects: Consent was obtained or waived by all participants in this study. Conflicts of interest: In compliance with the ICMJE uniform disclosure form, all authors declare the following: Payment/services info: All authors have declared that no financial support was received from any organization for the submitted work. Financial relationships: All authors have declared that they have no financial relationships at present or within the previous three years with any organizations that might have an interest in the submitted work. Other relationships: All authors have declared that there are no other relationships or activities that could appear to have influenced the submitted work.

\section{References}

1. Harapan H, Itoh N, Yufika A, et al.: Coronavirus disease 2019 (COVID-19): a literature review . J Infect Public Health. 2020, 13:667-73. 10.1016/j.jiph.2020.03.019

2. Yue H, Bai X, Wang J, et al.: Clinical characteristics of coronavirus disease 2019 in Gansu province, China . Ann Palliat Med. 2020, 9:1404-12. 10.21037/apm-20-887

3. Huang C, Wang Y, Li X, et al.: Clinical features of patients infected with 2019 novel coronavirus in Wuhan, China. Lancet. 2020, 395:497-506. 10.1016/S0140-6736(20)30183-5

4. Harapan H, Ryan M, Yohan B, et al.: Covid-19 and dengue: double punches for dengue-endemic countries in Asia. Rev Med Virol. 2021, 31:e2161. 10.1002/rmv.2161

5. Leisman DE, Deutschman CS, Legrand M: Facing COVID-19 in the ICU: vascular dysfunction, thrombosis, and dysregulated inflammation. Intensive Care Med. 2020, 46:1105-8. 10.1007/s00134-020-06059-6

6. Henrina J, Putra IC, Lawrensia S, Handoyono QF, Cahyadi A: Coronavirus disease of 2019: a mimicker of dengue infection?. SN Compr Clin Med. 2020, 2:1-11. 10.1007/\$42399-020-00364-3

7. Hernández-Fonseca JP, Durán A, Valero N, Mosquera J: Losartan and enalapril decrease viral absorption and interleukin 1 beta production by macrophages in an experimental dengue virus infection. Arch Virol. 2015, 160:2861-5. 10.1007/s00705-015-2581-1

8. Centers for Disease Control and Prevention: is it dengue or is it COVID-19? . (2020). Accessed: August 2, 2021: https://www.cdc.gov/dengue/healthcare-providers/dengue-or-covid.html.

9. Nacher M, Douine M, Gaillet M, et al.: Simultaneous dengue and COVID-19 epidemics: difficult days ahead? PLoS Negl Trop Dis. 2020, 14:e0008426. 10.1371/journal.pntd.0008426

10. Nath H, Mallick A, Roy S, Sukla S, Biswas S: Computational modelling supports that dengue virus envelope antibodies can bind to SARS-CoV-2 receptor binding sites: Is pre-exposure to dengue virus protective against COVID-19 severity?. Comput Struct Biotechnol J. 2021, 19:459-66. 10.1016/j.csbj.2020.12.037 